\title{
Etude Monte-Carlo du transport dans un gaz d'électrons bidimensionnel dégénéré
}

\author{
M. Mouis, P. Dollfus et R. Castagné \\ Institut d'Electronique Fondamentale, CNRS UA 22, Bat. 220, Université Paris-Sud, F-91405 Orsay Cedex, \\ France
}

(Reçu le $1^{\text {er }}$ juillet 1988, accepté le 3 octobre 1988)

\begin{abstract}
Résumé. - Pour simuler le transport dans le canal d'accumulation d'un transistor à effet de champ en régime de forte accumulation, nous avons mis au point un logiciel de simulation Monte-Carlo à champ constant tenant compte du caractère bidimensionnel du gaz d'électrons. Ce modèle inclut la quantification de la bande de conduction ; il utilise les interactions calculées numériquement après résolution de l'équation de Schrödinger. C'est d'autre part le premier modèle Monte-Carlo du gaz 2D qui prenne en compte le principe d'exclusion de Pauli. Nous présentons les résultats obtenus en fonction de l'intensité du confinement, pour 2 températures et selon que l'on tient compte ou non du principe d'exclusion.
\end{abstract}

\begin{abstract}
In order to simulate electron transport in the channel of a field-effect transistor in the strong accumulation regime, we have developped a Monte-Carlo particle model in the two-dimensionnal electron gas. We account for the conduction band quantization. The interactions are derived numerically after solving Schrödinger equation. Moreover, this is the first Monte-Carlo model taking into account the Pauli exclusion principle. We present the results for different confining field strengths, for two temperatures (300 and $77 \mathrm{~K}$ ) and with or without account for the Pauli exclusion principle.
\end{abstract}

\section{Introduction.}

Les dispositifs à effet de champ à haute mobilité offrent des perspectives très intéressantes pour les circuits intégrés rapides et de nombreuses structures utilisant le transport au voisinage d'une hétérojonction ou dans des puits quantiques sont actuellement en cours d'étude.

L'intérêt de ces dispositifs provient en premier lieu de la rapidité du transport électronique : le canal de conduction est localisé dans une zone très peu dopée, et au voisinage d'une hétérojonction réalisant une interface de confinement quasi idéale. Cependant, le confinement est tel, qu'une partie du canal est généralement constituée d'un gaz d'électrons bidimensionnel. C'est-à-dire que la bande de conduction se décompose en une superposition de sous-bandes quantifiées, que les électrons ne disposent plus que de 2 degrés de liberté dans chacune de ces sous-bandes et que le transport (en particulier les interactions électrons-défauts du matériau) est modifié. Ces différentes particularités jouent certainement des rôles d'importance inégale vis-à-vis du fonctionnement d'un dispositif.
D'autre part, pour obtenir un courant suffisamment élevé, on tend à fonctionner en régime de très forte accumulation, amenant le matériau au-delà du seuil de dégénérescence dans le canal. Il est donc nécessaire de juger l'importance du principe d'exclusion de Pauli dans un gaz bidimensionnel. Le principe d'exclusion peut bien sûr modifier les propriétés macroscopiques du transport. Il risque surtout de favoriser le transfert spatial des électrons au-dessus de la barrière interfaciale : en modifiant la distribution énergétique des porteurs, le principe d'exclusion de Pauli peut modifier de façon significative l'injection des porteurs au-dessus de cette barrière.

La simulation du transport dans un gaz bidimensionnel dégénéré peut apporter une aide précieuse pour évaluer l'impact du confinement et de la dégénérescence sur le fonctionnement des transistors à effet de champ (TEC) à hétérojonction.

\section{Le modèle.}

La simulation exacte du transport en gaz 2D est difficile. En effet, les probabilités de transition entre 
états dépendent de la forme du puits puisque les fonctions d'onde en dépendent. Simultanément, la forme du puits est fonction de la répartition de la densité d'électrons $n(z)$ perpendiculairement à l'interface ; elle peut donc varier selon l'intensité du champ électromoteur parallèle à l'interface $\left(E_{\mathrm{l}}\right)$ qui, en chauffant les électrons, modifie leur répartition sur les niveaux énergétiques dans le puits. L'étude rigoureuse demande la résolution simultanée de 3 équations couplées : l'équation de Schrödinger, l'équation de Poisson, l'équation de Boltzmann.

La résolution de chacune de ces équations est longue (la description du transport demande en particulier de nombreuses intégrations numériques), de sorte qu'il est très difficile de décrire exactement le transport en gaz bidimensionnel avec les moyens informatiques habituels. Moyennant un certain nombre de simplifications, nous avons mis au point un logiciel Monte-Carlo à $N$ particules qui permet de décrire le transport en gaz bidimensionnel dans un puits de potentiel quelconque.

Cette méthode permet d'étudier le gaz d'électrons hors de l'équilibre thermodynamique, par application d'un champ électromoteur $E_{l}$ (parallèle à l'interface). Ce champ peut être maintenu constant pour étudier les phénomènes stationnaires. L'application d'échelons temporels de champ électrique pourra permettre d'étudier ultérieurement les régimes de transport non stationnaire.

Les interactions sont décrites à partir des fonctions d'ondes électroniques, obtenues par résolution de l'équation de Schrödinger dans le puits. Cette étude ayant été essentiellement vouée à l'origine à l'étude du transport dans les HEMT GaAs-GaAlAs, nous avons considéré que l'utilisation de matériaux non dopés (zones actives, spacer) ainsi que la température habituelle de fonctionnement $(T \geqslant 77 \mathrm{~K})$ permettait de ne conserver que les interactions entre un électron et les phonons optiques polaires et acoustiques. Les interactions peuvent donner lieu à un changement de sous-bande ou non. Elles sont considérées comme inélastiques. Ceci est vrai également pour les interactions avec les phonons acoustiques: bien que l'échange d'énergie soit faible, il est important de le prendre en compte, tout au moins à très faible champ et à basse température, puisque c'est alors le seul mécanisme de relaxation de l'énergie. D'autre part notre étude se limite au transport à faible champ (c'est-à-dire pour des champs inférieurs au champ critique où apparaît le transfert vers les vallées latérales de la bande de conduction). Au-delà, les électrons retrouvent progressivement leur caractère 3D au fur et à mesure de leur transfert énergétique.

Par rapport à l'étude rigoureuse, la principale simplification consiste à conserver une forme de puits imposée, indépendante de la répartition des électrons dans les sous-bandes : $V(z)$ est un potentiel
« extérieur ». Pour pouvoir comparer de façon satisfaisante les résultats obtenus pour des densités surfaciques d'électrons différentes ou pour des températures différentes, nous avons choisi pour $V(z)$ le potentiel qu'on obtiendrait dans un puits d'accumulation tridimensionnel correspondant à la même densité surfacique. $V(z)$ est donc obtenu par résolution de l'équation de Poisson unidimensionnelle (perpendiculairement à l'interface), avec une statistique de Fermi-Dirac tridimensionnelle. Nous avons d'ailleurs vérifié que les positions énergétiques des sous-bandes calculées ainsi sont très proches de celles que donne une résolution self-consistante à l'équilibre thermodynamique (Fig. 1) [1, 2].

\begin{tabular}{cccc}
\hline $\begin{array}{c}\text { Méthode } \rightarrow \\
T(\mathrm{~K}) \\
\downarrow\end{array}$ & $\begin{array}{c}\text { Approximation } \\
\text { Poisson/1DG-3DK } \\
(\mathrm{meV})\end{array}$ & $\begin{array}{c}\text { Calculs } \\
\text { self-consistants } \\
(\mathrm{meV})\end{array}$ \\
\hline & & Ref. [2] & Ref. [1] \\
\cline { 2 - 4 } & & 50,3 & \\
& 57,4 & 85,9 & \\
& 87,7 & 107,7 & \\
& 109,3 & 124,1 & \\
& - & 137,9 & \\
$77 \mathrm{~K}$ & - & 47,5 & 53 \\
& 49,5 & 76,2 & 80 \\
& 74,4 & 93,5 & 94 \\
\hline
\end{tabular}

Fig. 1. - Comparaison entre les positions énergétiques des sous-bandes calculées dans notre approximation et les positions calculées par une méthode self-consistante à l'équilibre thermodynamique. La densité surfacique de charges $\left(n_{\mathrm{s}}=5 \times 10^{11} \mathrm{~cm}^{-2}\right)$ et le dopage $\left(N_{\mathrm{A}}=\right.$ $10^{15} \mathrm{~cm}^{-3}$ ) sont les mêmes.

[Comparison between the calculated subbands energy using our approximation or using a self-consistent method, at thermal equilibrium. The same surface charge density $n_{\mathrm{s}}=5 \times 10^{11} \mathrm{~cm}^{-2}$ and the same doping level $N_{\mathrm{A}}=$ $10^{15} \mathrm{~cm}^{-3}$ are used.]

Le logiciel a été validé en partie par les résultats obtenus en champ faible dans les gaz d'électrons non dégénérés. A $300 \mathrm{~K}$, on s'attend alors à ce que la distribution des électrons dans chaque sous-bande corresponde à une maxwellienne à 2 dimensions. De fait, la distribution énergétique dans chaque sousbande suit la distribution exponentiellement décroissante prévue théoriquement avec une statistique de Boltzmann et une densité d'états constante (Fig. 2a). Les taux d'occupation calculés dans chaque sous-bande ont la valeur théorique attendue. Enfin, la figure $2 \mathrm{~b}$ permet de vérifier l'accord entre la distribution des électrons en vitesse obtenue par la simulation et la distribution maxwellienne théorique. 


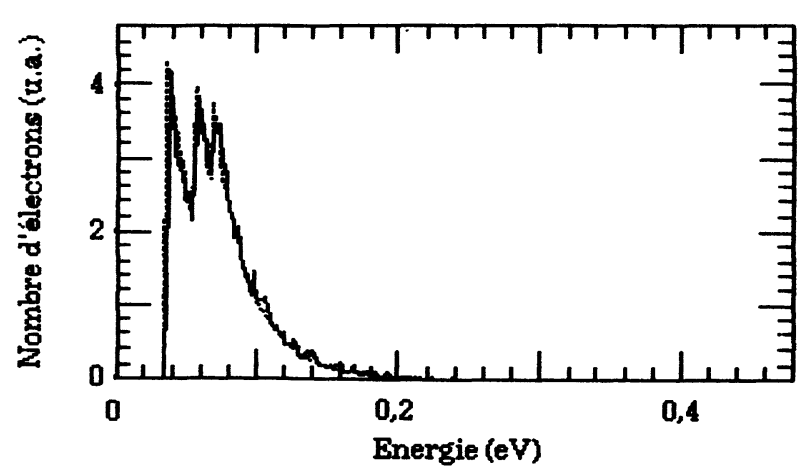

a)

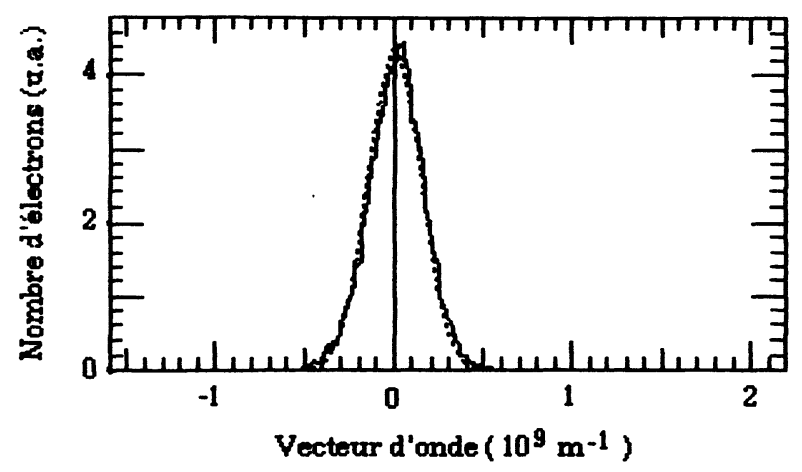

b)

Fig. 2. - Distribution des électrons en énergie (a) et en vitesse (b) calculées sous faible champ électromoteur $\left(E_{1}=0,5 \mathrm{kV} / \mathrm{cm}\right)$ à $T=300 \mathrm{~K}$. On a considéré 3 sousbandes. Les courbes en pointillés sont des distributions maxwelliennes à $300 \mathrm{~K}$.

[Calculated energy spectrum (a) and velocity spectrum (b) for a low heating field $\left(E_{1}=0.5 \mathrm{kV} / \mathrm{cm}\right)$ at room temperature. Three subbands are considered. The dotted curves are the theoretical Maxwellian distribution functions.]

\section{Propriétés de transport à $300 \mathrm{~K}$.}

La simulation permet d'obtenir les valeurs moyennes d'un certain nombre de grandeurs caractéristiques du transport (vitesse, mobilité, énergie cinétique) ainsi que la répartition des électrons sur les niveaux d'énergie et la valeur moyenne de l'énergie potentielle qui en résulte. Nous avons comparé les résultats pour deux valeurs de la densité surfacique d'électrons accumulés dans le canal $\left(n_{\mathrm{s}}=2 \times 10^{11}\right.$ et $7 \times 10^{11} \mathrm{~cm}^{-2}$ ).

Le calcul de la distribution énergétique des électrons permet de déterminer le champ critique audelà duquel les électrons commencent à transférer vers les vallées latérales de la bande de conduction. Ce champ est de l'ordre de $3 \mathrm{kV} / \mathrm{cm}$ pour $n_{\mathrm{s}}=$ $2 \times 10^{11} \mathrm{~cm}^{-2}$ et décroît légèrement lorsque le confinement augmente.

La figure 3 montre l'évolution de la vitesse moyenne avec le champ électromoteur. Un confinement plus important a plutôt tendance à dégrader la

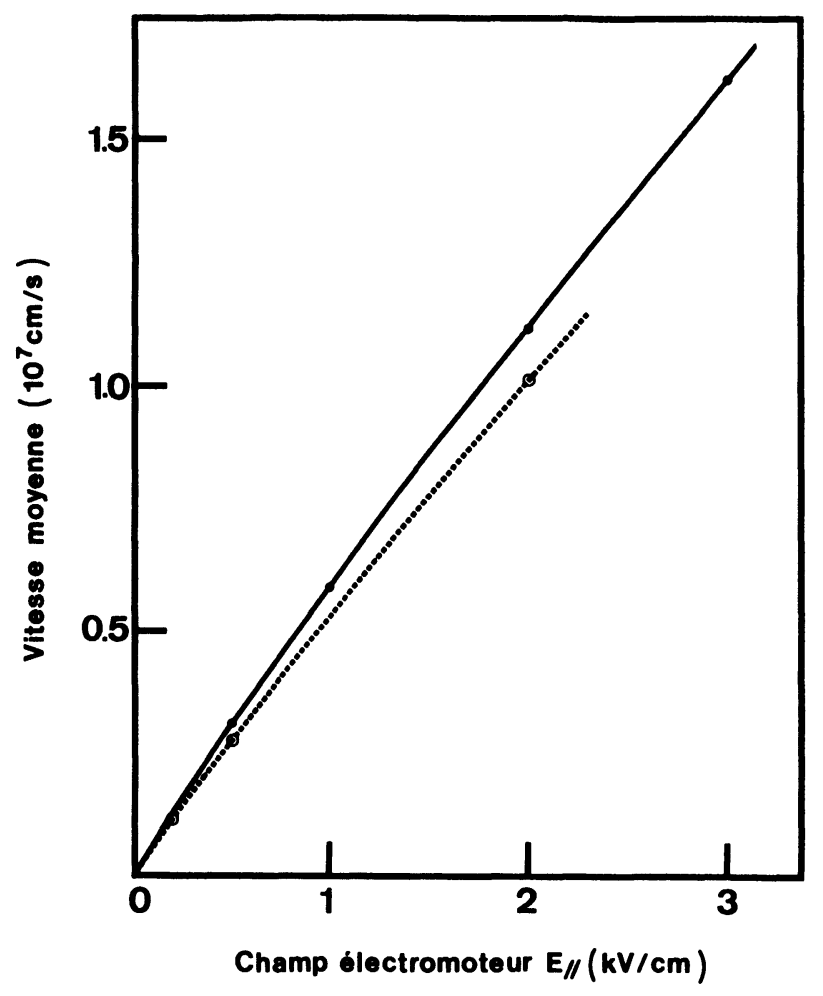

Fig. 3. - Vitesse moyenne à $300 \mathrm{~K}$ en fonction du champ électromoteur $E_{\mathrm{l}}$, pour deux intensités de confinement correspondant à des densités surfaciques d'électrons $n_{\mathrm{s}}=2 \times 10^{11} \mathrm{~cm}^{-2}$ (traits pleins) et $n_{\mathrm{s}}=7 \times 10^{11} \mathrm{~cm}^{-2}$ (tirets).

[Mean velocity at $300 \mathrm{~K}$ versus heating field for two confining field strengths, corresponding to the surface charge densities $n_{\mathrm{s}}=2 \times 10^{11} \mathrm{~cm}^{-2}$ (solid lines) and $n_{\mathrm{s}}=7 \times 10^{11} \mathrm{~cm}^{-2}$ (dashed lines).]

mobilité faible champ (elle passe de 6500 à $\left.6200 \mathrm{~cm}^{2} \mathrm{~V}^{-1} \mathrm{~s}^{-1}\right)$. Cet effet est cohérent avec ce qui peut être prévu dans un matériau n.i.d. puisque la probabilité d'interaction électron-phonon croît lorsque le confinement augmente. La dégradation associée à ce phénomène reste cependant faible. Notons que dans la réalité, les interactions avec les impuretés éloignées (dans $\mathrm{GaAlAs}$ ) et avec les impuretés locales (dans GaAs) restent importantes $\mathrm{du}$ fait des valeurs des concentrations résiduelles des niveaux de dopage non compensés. Ces interactions, de nature coulombienne, sont d'autant moins efficaces que les électrons ont une vitesse élevée. Elles deviennent donc moins efficaces au fur et à mesure que le niveau de Fermi pénètre dans la bande de conduction. Ce mécanisme, qui reste prépondérant dans les dispositifs réels et provoque un accroissement de la mobilité avec $n_{\mathrm{s}}$, masque la dégradation de mobilité observée ici pour un cas idéal $[1,3]$.

Sur la figure 4, nous avons représenté l'évolution des énergies cinétique, potentielle et totale lorsque le champ électromoteur varie. On constate que l'énergie potentielle est quasiment indépendante de 


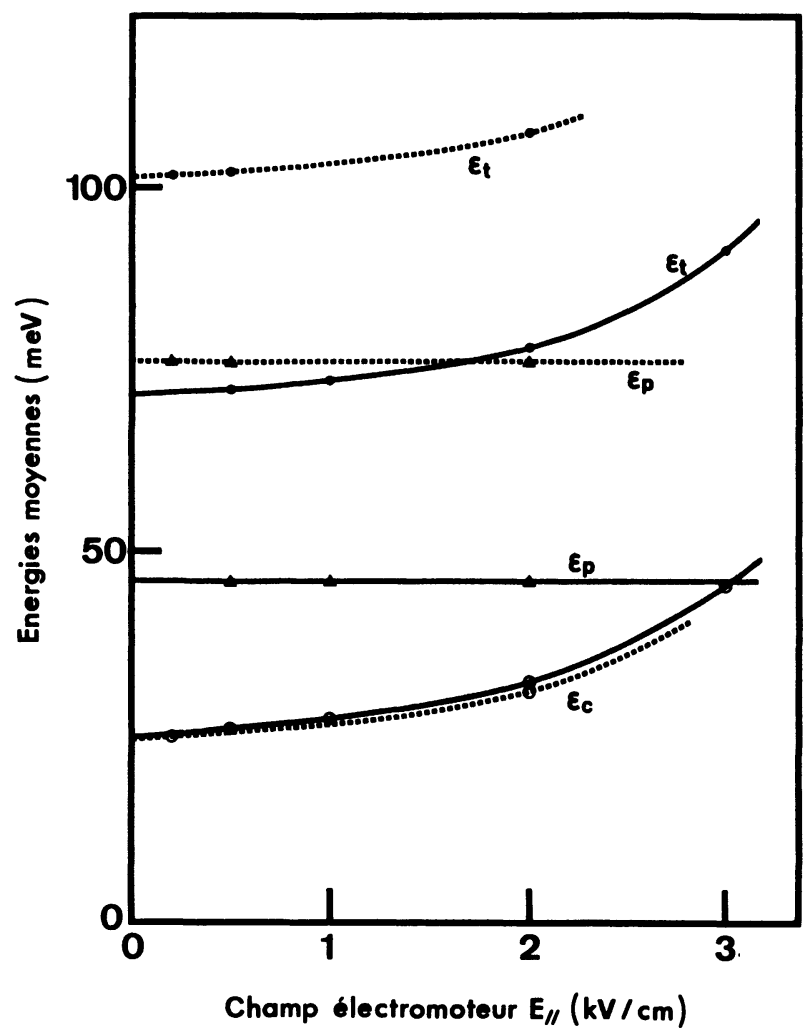

Fig. 4. - Evolution des énergies cinétique $\left(\varepsilon_{\mathrm{c}}\right)$, potentielle $\left(\varepsilon_{\mathrm{p}}\right)$ et totale $\left(\varepsilon_{\mathrm{t}}\right)$ à $300 \mathrm{~K}$, en fonction du champ électromoteur $E_{1}$, pour deux valeurs de la densité surfacique d'électrons : $n_{\mathrm{s}}=2 \times 10^{11} \mathrm{~cm}^{-2}$ (traits pleins) et $n_{\mathrm{s}}=7 \times 10^{11} \mathrm{~cm}^{-2}$ (tirets).

[Evolution of the kinetic energy $\left(\varepsilon_{\mathrm{c}}\right)$, of the potential energy $\left(\varepsilon_{\mathrm{p}}\right)$ and of the total energy $\left(\varepsilon_{\mathrm{t}}\right)$ versus the heating field $E_{1}$. The curves are given for two values of the surface charge density: $n_{\mathrm{s}}=2 \times 10^{11} \mathrm{~cm}^{2}$ (solid lines) and $n_{\mathrm{s}}=7 \times 10^{11} \mathrm{~cm}^{-2}$ (dashed lines) at $300 \mathrm{~K}$.]

$E_{\|}$; de fait, les taux d'occupation des sous-bandes restent sensiblement constants tant que $E_{\|}$reste inférieur au champ critique. Le gain d'énergie dans le champ $E_{\|}$se traduit uniquement par une augmentation de l'énergie cinétique, sans modification de la répartition entre les sous-bandes. On peut donc définir une température électronique commune à ces sous-bandes.

On constate que l'énergie cinétique est peu sensible aux modifications du confinement. Ce dernier provoque par contre une augmentation très importante de l'énergie potentielle moyenne. L'énergie totale est translatée d'autant et passe par exemple de $75 \mathrm{meV}$ à plus de $100 \mathrm{meV}$ en champ nul, lorsque $n_{\mathrm{s}}$ passe de $2 \times 10^{11}$ à $7 \times 10^{11} \mathrm{~cm}^{-2}$, alors qu'elle ne vaudrait que $40 \mathrm{meV}$ en volume.

L'intensité du confinement a donc des conséquences relativement réduites sur les paramètres macroscopiques du transport : mobilité et énergie cinétique sont peu affectées par une modification du champ de confinement. L'énergie potentielle des électrons y est par contre très sensible. Ceci peut amener l'énergie totale à des valeurs élevées, comparables à la discontinuité de la bande de conduction pour certaines hétérojonctions. Ceci peut favoriser le transfert spatial dans les zones fortement confinées des dispositifs (en particulier dans la zone d'accès de source). Ce mécanisme peut être responsable également d'une saturation de la transconductance dans les régimes de forte accumulation puisqu'il limite la densité surfacique d'électrons qui peuvent être accumulés dans le canal.

\section{Propriétés de transport à $77 \mathrm{~K}$.}

Les résultats précédents ont été complétés par une étude à plus basse température $(T=77 \mathrm{~K})$. Les phénomènes liés au caractère bidimensionnel apparaissent alors plus clairement de sorte que les études théoriques et expérimentales publiées sont plus nombreuses et permettent des comparaisons.

La courbe $v\left(E_{1}\right)$ calculée à $77 \mathrm{~K}$ est reportée sur la figure 5. Comme la courbe $v(E)$ en volume (dans un matériau très pur), elle présente une non-linéarité marquée à faible champ, qui a été mise en évidence par d'autres simulations du transport en gaz 2D [4] et par des mesures [5]. Cette non-linéarité apparaît pour des champs électriques voisins de $0,5 \mathrm{kV} / \mathrm{cm}$, du même ordre de grandeur qu'en volume. Elle semble d'ailleurs être liée au même phénomène [6].

A champ électromoteur très faible, et du fait de la forte probabilité d'émission d'un phonon optique

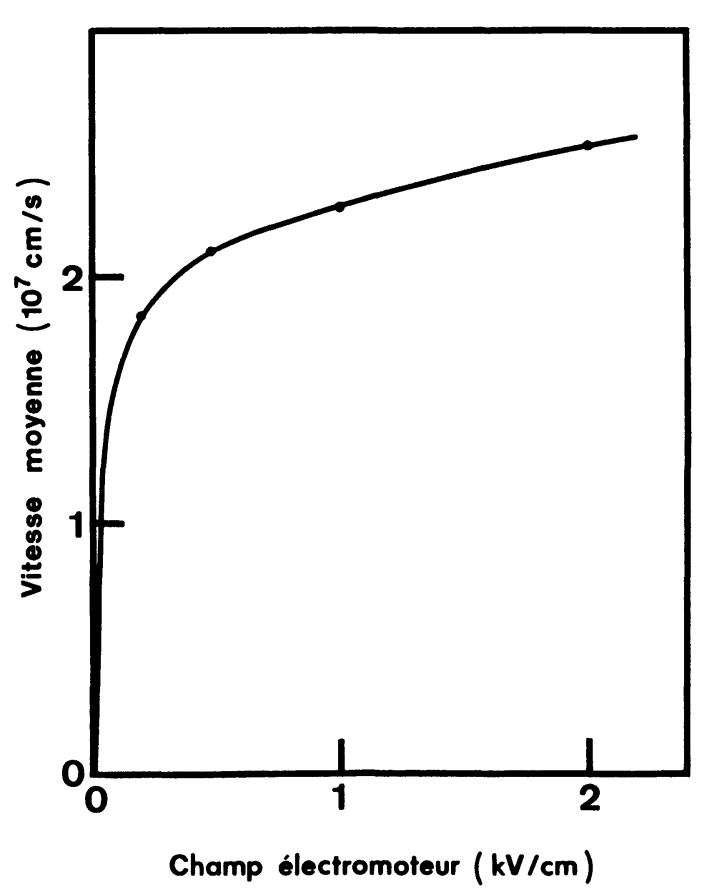

Fig. 5. - Evolution de la vitesse moyenne avec le champ électromoteur à $77 \mathrm{~K}$.

[Mean velocity at $77 \mathrm{~K}$ versus the parallel heating field.] 
polaire $\left(7 \times 10^{12} \mathrm{~s}^{-1}\right)$ par rapport aux autres interactions $\left(3 \times 10^{10} \mathrm{~s}^{-1}\right.$ pour les absorptions à même énergie), les niveaux énergétiques correspondant à une énergie cinétique supérieure à $\hbar \omega_{0}$ sont dépeuplés (Fig. 6a). La fréquence d'émission des phonons optiques polaires en régime stationnaire est donc relativement faible, faute d'électrons susceptibles de la subir.

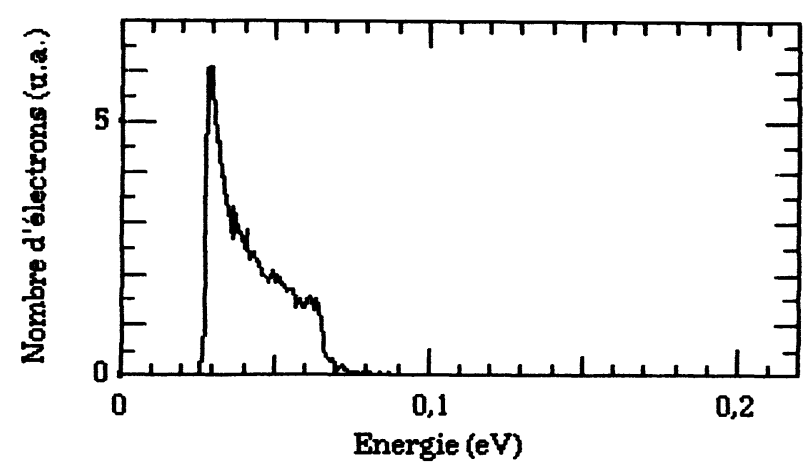

a)

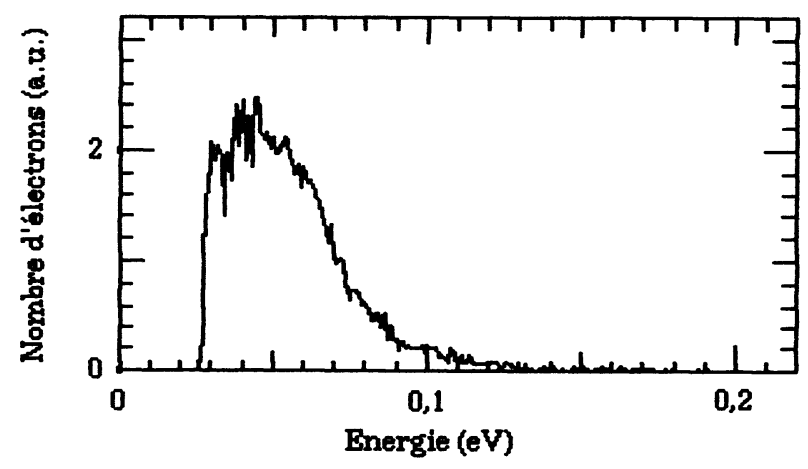

b)

Fig. 6. - Distributions énergétiques des électrons a) à faible champ $(0,2 \mathrm{kV} / \mathrm{cm})$ et b) à champ plus élevé $(2 \mathrm{kV} / \mathrm{cm})$, calculées pour $T=77 \mathrm{~K}$.

[Energy spectrum at $77 \mathrm{~K}$ for a) a low heating field $(0.2$ $\mathrm{kV} / \mathrm{cm})$ and $\mathrm{b}$ ) a stronger field $(2 \mathrm{kV} / \mathrm{cm})$.]

Par contre, lorsque le champ électromoteur augmente, la simulation montre une augmentation rapide de la fréquence des émissions de phonons optiques : le champ électrique devient suffisant pour que les électrons atteignent au cours de leur vol libre, des énergies supérieures à $\hbar \omega_{0}$ et soient ainsi susceptibles d'émettre un phonon. C'est ce qu'on observe sur les distributions énergétiques de la figure $6 \mathrm{~b}$. Cette augmentation de la fréquence des interactions se traduit par une réduction de la mobilité des électrons : on passe d'une mobilité très élevée, limitée par les phonons acoustiques $\left(E_{\|}<\right.$ $0,5 \mathrm{kV} / \mathrm{cm}$ ), à une mobilité plus faible, limitée par les phonons optiques polaires $\left(E_{\|}>0,5 \mathrm{kV} / \mathrm{cm}\right)$. La non-linéarité de la courbe $v\left(E_{\|}\right)$peut donc être attribuée, comme en volume, à l'apparition des interactions avec les phonons optiques polaires.

On calcule une mobilité $\mu=95000 \mathrm{~cm}^{2} \mathrm{~V}^{-1}$ $\mathrm{s}^{-1}$ pour $E_{\|}=0,2 \mathrm{kV} / \mathrm{cm}$. Cette mobilité varie très rapidement avec $E_{\|}$, de sorte que les comparaisons avec les mobilités de volume (de l'ordre de $2 \times$ $10^{5} \mathrm{~cm}^{2} \mathrm{~V}^{-1} \mathrm{~s}^{-1}$ à $77 \mathrm{~K}$ ) ou avec d'autres résultats sont en fait difficiles.

\section{Principe d'exclusion de Pauli.}

Le principe d'exclusion de Pauli intervient dans le calcul des probabilités d'interaction $\lambda(\varepsilon)$ puisqu'on utilise pour ce calcul la probabilité de transition entre deux états quelconques supposés libres et les fonctions d'occupation de ces états.

Lorsque le gaz est non dégénéré, tous les états finals sont libres et les probabilités d'interaction peuvent être calculées à l'avance (condition indispensable pour que la simulation du transport puisse être réalisée ensuite dans des temps de calcul raisonnables). Ce n'est plus le cas lorsque le gaz est dégénéré et il faut tenir compte de la fonction d'occupation réelle des états, à un instant donné. On peut envisager plusieurs solutions; nous avons choisi d'utiliser une méthode de réjection, plus compatible avec la philosophie des simulations Monte-Carlo et qui a été proposée également par Bosi pour étudier le transport en volume dans GaAs dégénéré [7]. Un certain nombre de précautions concernant le nombre d'électrons et les pas de discrétisation de l'espace réciproque doivent être observées pour obtenir un résultat correct $[8,9]$.

A température ambiante, la prise en compte du principe d'exclusion de Pauli perturbe très peu les résultats. Son effet est plus sensible à $77 \mathrm{~K}$.

En limitant le nombre d'états disponibles, l'application du principe d'exclusion de Pauli a pour effet de réduire le nombre d'électrons vers le minimum énergétique des sous-bandes (où la fonction de distribution est maximale). De la même manière, le principe d'exclusion de Pauli a pour effet de réduire le taux d'occupation de la sous-bande fondamentale au profit des sous-bandes excitées (Fig. 7). Cette redistribution des électrons se traduit par une très légère augmentation de l'énergie potentielle des électrons.

La seconde conséquence notable consiste en une réduction de la non-linéarité de la courbe $v\left(E_{\|}\right)$. Ce phénomène peut être interprété de la façon suivante : le principe d'exclusion de Pauli étant surtout efficace en bas de sous-bande, son effet est de reporter une partie des électrons à des énergies plus élevées dans chaque sous-bande; ceci se traduit d'ailleurs par une augmentation de l'énergie cinétique moyenne de la population.

Le nombre d'électrons d'énergie supérieure à $\hbar \omega_{0}$ est plus important et la fréquence des émissions 


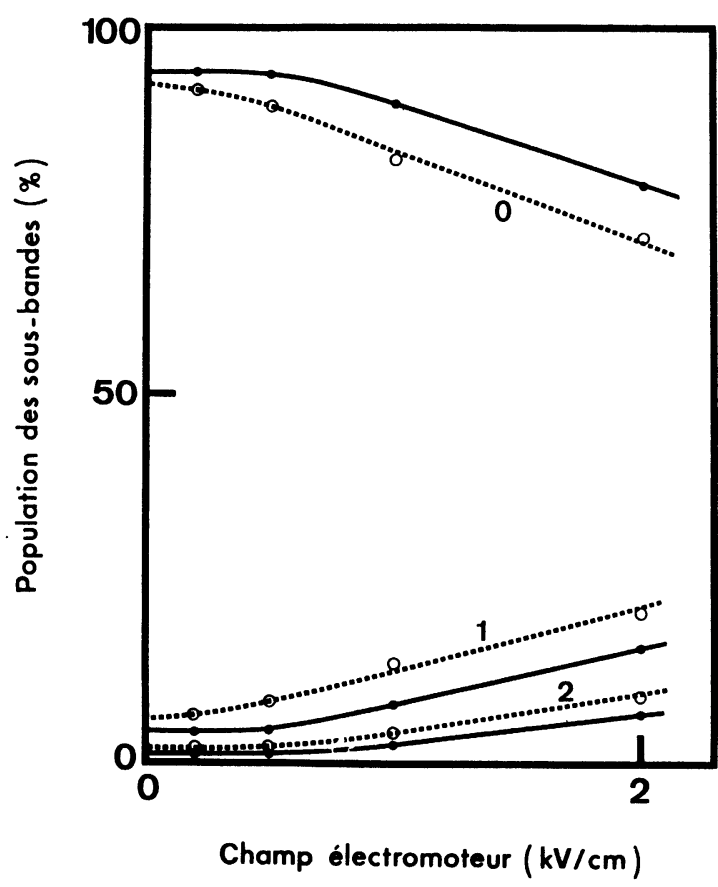

Fig. 7. - Taux d'occupation des sous-bandes, calculés en fonction du champ électromoteur, selon que l'on prend en compte le principe d'exclusion de Pauli (tirets) ou non (traits pleins). La température est $T=77 \mathrm{~K}$.

[Sub-band population versus the parallel heating field with account for the Pauli exclusion principle (dashed lines) or not (solid lines). The temperature is $T=77 \mathrm{~K}$.]

de phonons optiques polaires enregistrée dans la simulation (pour un même champ $E_{\|}$) s'accroît d'autant. Même si une partie de ces interactions est rendue impossible du fait de l'occupation éventuelle de l'état final, le bilan conduit à une dégradation plus rapide de la mobilité. Cet écart disparaît cependant dès que le chauffage dû au champ électromoteur devient assez efficace et les propriétés de transport ainsi que les distributions énergétiques deviennent identiques.

\section{Conclusion.}

En conclusion, les propriétés de transport globales à $300 \mathrm{~K}$ (vitesse, mobilité et énergie cinétique moyennes) semblent sensiblement les mêmes qu'en volume.

Le confinement a comme principal effet de provoquer une augmentation de l'énergie potentielle de la population, qui peut favoriser un transfert vers les vallées latérales ou vers le matériau à grand gap. Cette augmentation de l'énergie potentielle n'est importante que si les énergies au bas des sousbandes sont grandes par rapport à l'énergie thermique. Elle sera donc surtout sensible dans la zone d'accès de source ou, dans les régimes de très forte accumulation, sous la grille, provoquant une saturation de la transconductance du dispositif. Dans le reste du dispositif, le champ interfacial est trop faible ou bien la population électronique est trop chaude pour qu'on puisse la considérer comme bidimensionnelle.

Le caractère bidimensionnel du gaz d'électrons disparaît pour des champs électromoteurs relativement faibles par rapport aux champs électriques couramment rencontrés dans les dispositifs courts.

Enfin, le principe d'exclusion de Pauli est pratiquement sans influence à température ambiante et à $77 \mathrm{~K}$, il ne joue un rôle sensible que pour des champs électromoteurs faibles $(<2 \mathrm{kV} / \mathrm{cm}$ pour $n_{\mathrm{s}}=2 \times 10^{11} \mathrm{~cm}^{-2}$ ).

\section{Bibliographie}

[1] ZimmermanN, J.; Yen, W., Etude de la dynamique des électrons à deux dimensions dans les hétérojonctions, Rev. Phys. Appl. 22 (1987) 1501-1513.

[2] Yokoyama, K., Hess, K., High field transient transport in modulation doped heterostructures, Phys. Rev. B 33 (1986) 5595-5606.

[3] Hirakawa, K., SaKaki, H., Mobility of the twodimensional electron gas at selectively doped $\mathrm{n}$ type $\mathrm{Al}_{x} \mathrm{Ga}_{1-x} \mathrm{As} / \mathrm{GaAs}$ hererojunctions with controlled electron concentrations, Phys. Rev. B 33 (1986) 8291-8303.

[4] Tomizawa, M., YoshiI, A., Yokoyama, K., Modeling for an $\mathrm{AlGaAs} / \mathrm{GaAs}$ heterostructure device using Monte-Carlo simulation, IEEE Electron. Device Lett. EDL 6 (1985) 332.

[5] Masselink, W. T., Braslau, N., La Tulipe, D., WANG, W. I., WRIGHT, S. L., Velocity-field characteristics of carriers in $\mathrm{AlGaAs} / \mathrm{GaAs}$ modulation doped structures, 14th Symposium on GaAs and Related Compounds (Heraklion, Crête) 1987.

[6] Riddoch, F. A., Ridley, B. K., J. Phys. C : SolidState Phys. 16 (1983) 6971.

[7] Bosi, S., JACOBONI, C., Monte-Carlo high field transport in degenerate GaAs, J. Phys. C : Solid-State Phys. 9 (1976) 315.

[8] Mouis, M., Etude théorique du fonctionnement des dispositifs à effet de champ haute mobilité à hétérojonction, Thèse d'état, Université ParisSud (1988).

[9] Lugli, P., FerRY, D. K., Degeneracy in the Ensemble Monte-Carlo method for high field transport in semiconductors, IEEE Trans. on Electron Devices, ED 32 (1985) 2431. 